\title{
Electromagnetic Interference Shielding Response and Rheological Behavior of Lightweight Nanocomposites based on isotactic Polypropylene and Al Nanoparticles
}

Enrique Blázquez-Blázquez ${ }^{1}$, Javier Arranz-Andrés ${ }^{1}$, Jorge Ressia ${ }^{2,3}$, Enrique M. Vallés ${ }^{2}$, Pilar Marín ${ }^{4}$, Ana M. Aragón ${ }^{4}$, Ernesto Pérez ${ }^{1}$, María L. Cerrada ${ }^{1 *}$

${ }^{1}$ Instituto de Ciencia y Tecnología de Polímeros (ICTP-CSIC). Juan de la Cierva 3, 28006 Madrid, Spain.

${ }^{2}$ Planta Piloto de Ingeniería Química - PLAPIQUI (UNS-CONICET). Camino La Carrindanga Km 7, 8000 Bahía Blanca, Argentina.

${ }^{3}$ Comisión de Investigaciones Científicas de la Provincia de Buenos Aires (CIC). La Plata, Argentina.

${ }^{4}$ Instituto de Magnetismo Aplicado (UCM-ADIF-CSIC) and Departamento de Física de Materiales (UCM). P. O. Box 155, Las Rozas, 28230 Madrid, Spain.

Correspondence to: $\underline{\text { mlcerrada@ictp.csic.es }}$ 


\begin{abstract}
Nanocomposites based on isotactic polypropylene (iPP) and different content of $\mathrm{Al}$ nanoparticles have been prepared in order to gain knowledge of their electromagnetic interference (EMI) shielding capability. This potential has been analyzed from attenuation upon reflection measurements at microwave frequency range. Moreover, shielding characteristics have been checked by Attenuated Total Reflectance (ATR) and correlated to the ones achieved by X-ray diffraction with synchrotron radiation. Very promising results have been obtained, with an excellent balance between shielding efficiency and sample weight. Hence, these materials are potentially good alternatives to replace metals for this application, avoiding electromagnetic environmental pollution. The effect of incorporation of Al nanoparticles on the iPP processing properties has been additionally evaluated by a rheological study at a very broad frequency range.
\end{abstract}

Keywords: Attenuation upon reflection measurements; electromagnetic interference shielding; composite materials; storage shear modulus; melt viscosity. 


\section{Introduction}

Performance of every day more sophisticated electronic devices for industrial, commercial and military applications can be affected by electric and electronic signals emitted by other equipments. This is the reason why sources of electromagnetic interference (EMI) are considered as a feasible contemporary cause of environmental pollution. In addition to those adverse effects provoked by EMI on the current electronic devices [1-4] there is also an increasing concern about the long-term consequences on human health $[5,6]$ caused by the continuous exposure to those electric and magnetic fields.

Three mechanisms have been described to be involved in the EMI shielding: reflection, absorption and multiple-reflection [1,3,7,8]. For a sheet of homogeneous conductive material (not a composite of a conductive filler and insulating matrix), reflection is often the primary shielding mechanism $[1,8]$. Absorption is the second important mechanism and it depends on the thickness of the shield. Shielding by absorption is enhanced when the shielding material has electrical or magnetic dipoles interacting with the EM waves. The third shielding mechanism is multiple-reflection. It concerns the internal reflections within the shielding material. Typically, multiple-reflection decreases the overall shielding if the shield is thinner than the skin depth and can be ignored if the shield is thicker than the skin depth.

Metals and metallic compounds are the traditional EMI materials since they show an excellent shielding efficiency ascribed to their high electrical conductivity. A specific material can be used as an absorber or a reflector of the electromagnetic radiation [9] depending on its electromagnetic properties. The drawbacks of metals are related to their poor chemical resistance, high density, oxidation and corrosion as well as processing difficulties. Polymermatrix (nano)composites containing conductive fillers turn out an interesting alternative for shielding applications, to overcome those metal limitations, and allow the design and development of inert, durable and lightweight materials with the advantage of the much better processability of polymers and the high shielding efficiency of metal nanoparticles. Accordingly, the reduced costs as well as easiness of production are crucial for evaluating 
the potential of new shielding nanocomposites at industrial level. The latest is related to the fact that incorporation of very small concentrations of (nano)particles may alter the phase behavior and the flow properties in polymers. Thus, comprehension about how (nano)particles might affect either morphology or structure [10-12] as well as system dynamics [13-15] is a key factor in these polymer-nanoparticle hybrids to develop design rules for engineering materials with desired properties. And, a study of the rheological behavior of these polymer-metal nanocomposites turns out mandatory in order to learn about how processability of a given polymer will be affected by incorporation of a certain amount of metal (nano)particles.

Isotactic polypropylene (iPP) is a fast growing commodity resin since it has many desirable and useful physical properties [16]. Furthermore, its versatility at industrial level is also directly associated with its relatively low manufacturing cost. These characteristics promote its use in a wide variety of applications, which are in continuous growth since iPP becomes competitive with other more expensive engineering plastics. Incorporation of specific fillers [17-19] to enhance some of those excellent properties can allow spreading out even more its applications. For instance, it was reported that iPP-copper composites exhibited a promising attenuation upon transmission response at $2 \mathrm{EHz}$ [20]. The utilization of cooper composites may lead to shielding materials with a significant reduction in weight when compared with classic metals and metallic compounds. Nevertheless, the increasing market price of copper presents some inconveniences. As an alternative, lighter and cheaper Al nanoparticles were employed to obtain iPP-Al (nano)composites [21]. They showed up to a $99 \%$ attenuation of the electromagnetic radiation at the same frequency (2 EHz). Other composites based on iPP for EMI shielding applications were reported in literature at the radio frequency range. They incorporated carbon black [22,23], carbon nanotubes [24,25], carbon nanofibres [26] and graphite [27,28]. Carbonaceous fillers and stainless steel fibers were also evaluated for this purpose [29]. 
The main purpose of this article is twofold: firstly, to study the EMI response of lightweight nanocomposites based on isotactic polypropylene and Al nanoparticles at the microwave frequency. Secondly, to investigate how the iPP processability might be affected by incorporation of $\mathrm{Al}$ nanoparticles at those compositions able to be industrially processed. Therefore, the effect of aluminum nanoparticles on the iPP rheological behavior at distinct contents will be tested at different temperatures to learn if a master curve can be built to predict global viscoelastic behavior on molten state, i.e., their thermo-rheological simplicity. This evaluation is of great importance since these hybrid materials are prepared by melt mixing, providing an idea of their final processability at large scale. The EMI behavior of these polymer-metal nanocomposites will be also checked at an infrared frequency to know if this technique can be used as a preliminary tool for determining the shielding capabilities of these materials against electromagnetic radiation. This electromagnetic response will be as well compared with that observed at $2 \mathrm{EHz}$.

\section{Experimental Part}

\section{Materials}

A commercially available metallocene-catalyzed isotactic polypropylene (Metocene HM562P: melt flow index of $15 \mathrm{~g} / 10 \mathrm{~min}$-ISO 1133-; melting temperature $\left(\mathrm{T}_{\mathrm{m}}\right)$ of $146{ }^{\circ} \mathrm{C}$, kindly supplied by LyondellBasell) was selected in this work as polymeric matrix. Aluminum nanoparticles, with an average particle size of $18 \mathrm{~nm}$, were purchased from IOLITEC.

\section{(Nano)composites and film preparation}

Composites with different contents in Al nanoparticles (1, 5, 10, 20, 30\% in volume), labeled as $\mathrm{Al} 1, \mathrm{Al} 5, \mathrm{Al} 10, \mathrm{Al} 20$ and $\mathrm{Al} 30$ respectively, were prepared through melt processing at $165^{\circ} \mathrm{C}$ and at $120 \mathrm{rpm}$ for $40 \mathrm{~min}$ in a Haake Minilab (Thermo Electron Corporation) twinscrew extruder with a volumetric capacity of $7 \mathrm{~cm}^{3}$ using corotating conical screws.

After extrusion, films with thickness around 250 microns were processed by compression molding in a Collin press between hot plates $\left(190^{\circ} \mathrm{C}\right)$ at a pressure of $10 \mathrm{MPa}$ 
for $6 \mathrm{~min}$. A relatively fast cooling (of about $80^{\circ} \mathrm{C} / \mathrm{min}$ ) was applied to the different films within the press refrigerated with cold water from the melt to room temperature. The same procedure was applied to obtain iPP films without Al nanoparticles in order compare the performance of the different composites with the behavior of the pristine iPP.

\section{Sample Characterization}

The composite density, $\rho$, was determined via the hydrostatic weighing method [30]. The apparent weight Wi of a specimen is measured in two different media, e.g. air and water, and the density was obtained from:

$$
\rho_{i}=\frac{W_{\text {air }}\left(\rho_{\text {water }}-0.0012\right)}{0.99983\left(W_{\text {air }}-W_{\text {water }}\right)}+0.0012
$$

where $\rho_{\text {water }}\left(0.99732 \mathrm{~g} / \mathrm{cm}^{3}\right.$ at $\left.24.0^{\circ} \mathrm{C}\right)$ and $\rho_{\text {air }}\left(0.0012 \mathrm{~g} / \mathrm{cm}^{3}\right)$ are water and air densities, respectively, and 0.99983 is a geometric balance correction. All measurements were performed on a Sartorius balance; model Kern 770 , with a resolution of $\pm 0.1 \mathrm{mg}$. The average density was obtained with at least three samples per composite (each sample was also measured twice). Polypropylene and aluminum densities are 0.9 and $2.7 \mathrm{~g} / \mathrm{cm}^{3}$, respectively. The results obtained for the different nanocomposites were: $0.92,0.99,1.05$, 1.20 and $1.38 \mathrm{~g} / \mathrm{cm}^{3}$ for Al1, Al5, Al10, Al20 and Al30. These values point out the lightweight nature of the materials under study.

Scanning electron microscopy (SEM) experiments were carried out with a XL30 ESEM PHILIPS equipment. The samples were cryo-fractured prior to SEM observations.

The rheological characterization was carried out in small-amplitude oscillatory shear mode using a dynamic rotational rheometer AR-G2 from TA Instruments ${ }^{\circledR}$. The tests were performed using parallel plates $25 \mathrm{~mm}$ in diameter, at a frequency range between 0.1 and $100 \mathrm{rad} / \mathrm{s}$, and various temperatures ranging from 160 to $210^{\circ} \mathrm{C}$. All tests were carried out at small strains in order to assure the linearity [31] of the dynamic responses. To verify this, the 
series of frequency sweeps were repeated twice with the same sample at different strains. Excellent agreement between these results was found in all cases.

Stress relaxation tests in the linear region were additionally performed at $160{ }^{\circ} \mathrm{C}$ in order to expand the frequency response at lower frequencies. These measurements allow studying the behavior of the relaxation modulus as a function of time, $\mathrm{G}(\mathrm{t})$, over a longer time scale, providing information about the slowest dynamics of the nanocomposites. These data were subsequently transformed by means of the Rheology Advantage Data Analysis ${ }^{\circledR}$ software provided with the TA Instruments ${ }^{\circledR}$ rheometer. In this way, the frequency dependent shear elastic, $G^{\prime}(\omega)$, and loss, $G^{\prime \prime}(\omega)$, moduli were obtained in a range of frequencies that expands, at least, two decades below $0.1 \mathrm{rad} / \mathrm{s}$, which was the minimum frequency covered by the shear sinusoidal oscillations experiments.

Wide-angle X-ray diffraction experiments were carried out at room temperature in the transmission mode using synchrotron radiation (with $\lambda=0.150 \mathrm{~nm}$ ) for all the samples in the soft-condensed matter A2 beamline at DESY (Hamburg, Germany). A MARCCD detector, placed at $135 \mathrm{~mm}$ from the sample, was used. The calibration was performed with the crystalline diffractions of polyethylene terephthalate. The 2D X-ray diffractograms were processed using the A2tool program developed to support beamline A2 data processing. The transmission of the samples to this radiation was determined by measuring the relative intensities of the direct beam before and after the specimen (in the ionization chamber and in the beamstop, respectively).

The IR spectra were obtained on films using a Perkin Elmer FTIR spectrometer equipped with an ATR device, scanning between 600 and $4000 \mathrm{~cm}^{-1}$. Sixteen scans were accumulated for each specimen at a resolution of $4 \mathrm{~cm}^{-1}$.

The electrical properties were measured with a Novocontrol BDS system comprising a frequency response analyzer (Solartron Schlumberger FRA 1260) and a broadband dielectric converter with an active sample head. Gold disk electrodes (20 $\mathrm{mm}$ in diameter) 
were used in the dielectric measurements carried out at $25^{\circ} \mathrm{C}$ in the frequency window $10^{-2}$ to $10^{7} \mathrm{~Hz}$. The equipment was adjusted with a $100 \Omega$ calibration standard.

An anechoic chamber was used to study electromagnetic behavior where two TEM horn antennas (EMCO3160-07) are placed in the far field (Fraunhofer region) of the absorbing material. Their bandwidth goes from 7 to $15 \mathrm{GHz}$. The absorbing material is placed in front of a metallic plate that ensures total reflection. The plate dimension is $25 \times 25$ $\mathrm{cm}^{2}$. They are surrounded by the same absorbers of the anechoic chamber to avoid border effects. A metallic sheet is covered in these experiments by a dielectric slab of thickness $d_{3}$ and a PP-Al based composite of thickness $d_{2}$ is located over them covering the assembly. Therefore, the electromagnetic radiation is incident on the composite slab, goes through it and the dielectric, and is reflected by the metallic surface [32]. The reflection coefficient at the surface of the composite slabs [33], $R_{0}$, depends on air, dielectric and composite impedances as well as on thickness of the dielectric and composite. Since the TEM horn antennas are in the far field, the waves can be considered planar waves propagating in TEM mode. The antennas are connected to a Network Analyzer (AgilentE8362BPNA Series Network Analyzer), and $S_{12}$ is measured, after careful calibration procedure based on open air measurements, where $S_{12}$ is the reverse transmission coefficient of $50 \mathrm{~W}$ terminated input. Both real and imaginary part of $R_{0}$ are measured and $R$ is represented and defined from the modulus, $\left|R_{0}\right|$, as

$$
R=20 \log \left(\left|R_{0}\right|\right)(d B)
$$

\section{Results and discussion}

\section{Rheological characterization}

The viscoelastic behavior in the molten state of PP/Al composites materials prepared by blending of Al nanoparticles within iPP matrix was investigated. Al nanoparticles instead of micrometric particles have been chosen as an attempt of boosting ultimate processability 
and of reducing Al content required for attaining lighter (and cheaper) materials with good physical properties as well as EMI shielding capability.

Dynamic frequency sweep tests and stress relaxation experiments were performed in the linear viscoelastic region to obtain information about the type of interactions that prevails between the iPP and the Al nanoparticles. Combination of these two types of measurements allows spreading the experimental frequency window in the low frequency range. Previous works suggested that the nature of the particle-polymer interactions (attractive vs. repulsive) plays an important role on the changes induced in the rheological characteristics of a molten polymeric matrix [34] when small amounts of nanofillers are incorporated [35-44]. If polymerparticle interactions are weak, the viscoelastic response of a polymer melt filled with low concentration of nanoparticles is very similar to that exhibited by the virgin polymer [35]. Nevertheless, a significant sluggish of the polymer dynamics has been reported in systems where significant attractive interactions exist. This feature was ascribed to a dramatic reduction in the mobility of adsorbed polymeric segments along the nanoparticle surface and association of nanoparticles into larger aggregates as a consequence of particle bridging by adsorbed polymer molecules $[35,36,39]$. In contrast, a strong decrease in viscosity has been found for silica filled polypropylene and ascribed to selective adsorption of high molar mass polymer chains on the filler [44].

Figure 1 shows the evolution of the elastic modulus, $G^{\prime}(\omega)$, and the loss modulus, G" $(\omega)$, as a function of frequency for the pristine iPP and the nanocomposites containing up to a 10 vol. \% of Al nanoparticles. A reference temperature of $160^{\circ} \mathrm{C}$ was chosen in these plots and all data collected at temperatures ranging from 170 to $200{ }^{\circ} \mathrm{C}$ under dynamic shear sinusoidal oscillation experiments were shifted to that reference temperature according to the time-temperature superposition principle in order to build master curves of the dynamic response. Good superposition of the data was obtained for these samples. Figure 1 also shows with open symbols the converted data from the stress relaxation experiments obtained at $160^{\circ} \mathrm{C}$. The superposition of the results from both experiments is fairly good and 
allows, as aforementioned, extending the width of the relaxation spectrum for almost three decades.
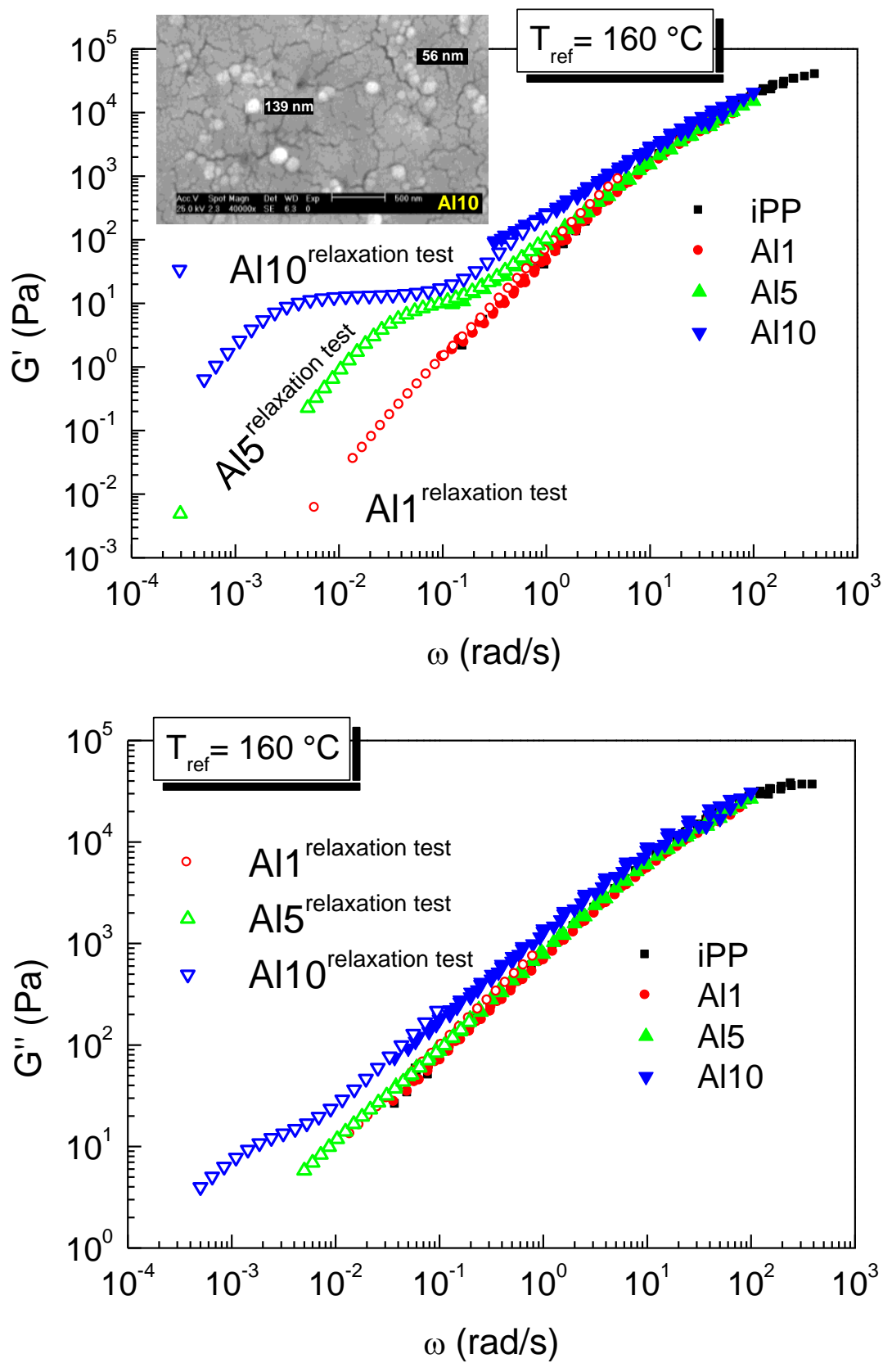

Figure 1. Reduced frequency dependence of storage modulus, $G^{\prime}(\omega)$ (upper plot) and loss modulus, G" $(\omega)$ (lower plot) for pristine iPP homopolymer and its Al1, Al5 and Al10 nanocomposites. A SEM picture for the Al10 nanocomposite is depicted as inset in the upper plot. Solid symbols represent results obtained from small-amplitude oscillatory shear experiments at a temperature interval; open symbols are referred to data achieved from stress relaxation measurements at $160^{\circ} \mathrm{C}$. 
A characteristic flow behavior for the pure iPP was found with power-laws for the frequency dependence in the terminal region of the elastic and loss moduli close to $G^{\prime}(\omega) \approx$ $\omega^{2}$ and $G^{\prime \prime}(\omega) \approx \omega$. Incorporation of a small amount of Al nanoparticles (Al1 hybrid) does not significantly affect the elastic modulus. Even when the extended relaxation behavior of the terminal zone is included with the incorporation of the transformed data from the stress relaxation experiments, no significant differences are observed between the pristine polymer and the Al1 nanocomposite. At very low frequencies, loss modulus values in the Al1 hybrid start, however, to slightly depart from those corresponding to pure iPP, denoting an increment in the dissipation modes induced by the presence of the nanofiller.

An increase in Al content from 1 to $5 \%$ in volume dramatically changes at low frequency the viscoelastic response of the Al5 melt compared with that shown by the neat iPP polymer. At frequencies slightly higher than $10 \mathrm{rad} / \mathrm{s}$, the viscoelastic response of Al5 is similar to that found in iPP. This is a clear indication that the short time response of the nanocomposite is ruled by the polymer matrix. A progressive departure of the elastic modulus of this nanocomposite from the pure iPP behavior is evident at the intermediate region of frequencies (between $0.3-10 \mathrm{rad} / \mathrm{s}$ ), demonstrating the existence of an elastic reinforcement effect due to the presence of Al particles. At lower frequencies where pristine iPP goes through its terminal relaxation zone, the Al5 nanocomposite exhibits a low frequency plateau that extends for about half a decade in frequency before entering in its terminal region. This behavior implies that the stress relaxation process of the melt is effectively restrained by the presence of the Al nanoparticles. The Al5 nanocomposite recovers the liquid-like properties with $G^{\prime}(\omega)$ dependence falling with a power-law exponent of 2 at frequencies below $0.02 \mathrm{rad} / \mathrm{s}$. This type of behavior has been explained as a consequence of the formation of a temporary network where nanoparticles are interconnected by the adsorbed polymer molecules, i.e. rheological percolation threshold exists [45]. At longer time scales, this network could finally be relaxed as the adsorbed polymeric molecules are released by different mechanisms [35]. 
The solid-like behavior at the low-frequency region is clearly noticeable from the results obtained from the Al10 nanocomposite, but now all the different zones of the viscoelastic spectra are more visibly defined. At the high frequency zone, the $\mathrm{G}^{\prime}$ dependence upon frequency is similar to that exhibited by pure iPP. This region is shifted to frequencies higher than $50 \mathrm{rad} / \mathrm{s}$ and the low frequency plateau now extends for more than one decade in frequency down to a frequency around $0.004 \mathrm{rad} / \mathrm{s}$ before entering into the terminal region. A noticeable increase in $\mathrm{G} "$ is also observed at this amount of nanofiller and at those low frequencies. The SEM picture inserted in the upper plot of Figure 1 shows for the Al10 material the evidence of a high degree of Al dispersion within iPP matrix. There are not metallic domains of large size across the section even at this high Al content. The average size of $\mathrm{Al}$ aggregates is ranging from around 55 to $140 \mathrm{~nm}$, fact that indicates that the preparation method has been rather efficient to distribute homogeneously the $\mathrm{Al}$ nanoparticles within the iPP and to lead to a good interfacial adhesion. The rheological results have clearly pointed out the formation of a rheological filler network and the morphological characteristics show the homogenous distribution of relatively small $\mathrm{Al}$ domains that allow the entrapment of polymeric chains within this filler network, which hinders the polymeric flow.

Some iPP based nanocomposites are reported to exhibit similar response to that here described [28] while in other materials the behavior is rather different $[29,46,47]$. Changes can be related to particle shape, size, state of dispersion and concentration. Each system is, then, singular. Aspect ratio of the filler has a very important role and the rheological percolation threshold can be reached at significantly lower filler content as aspect ratio increases. This is the case of iPP composites reinforced with carbon nanotubes (CNTs) in comparison with the Al based materials here evaluated, although distinct responses have been also described in those CNT based materials. For instance, no significant effect at low frequency was reported in composites where CNTs were directly dispersed in PP by meltblending [25]. Only, a small increase in the storage modulus started to be detected at CNTs 
content of 2 wt.\%. The influence of CNT on the rheological behavior was also very small when they were dispersed in PP by melt-blending in presence of $10 \mathrm{wt} . \%$ in polypropylenegraft-maleic anhydride (PP-g-MA) used as a compatibilizer. Dispersion led to a small improvement employing the coprecipitation technique but transition from liquid-like to solidlike viscoelastic behavior was not achieved. Nevertheless, the dispersion of CNTs in the presence of polypropylene-graft-aminomethylpyridine (PP-g-AMP) had a drastic effect on the rheological behavior of PP even at CNT loading as low as $1 \mathrm{wt} \%$. A plateau was observed at low frequencies, suggesting that the percolation threshold was close to be reached. This effect was even more pronounced when the CNTs were dispersed with the compatibilizer by coprecipitation [25]. A decrease in the terminal slopes was observed with increasing CNT loading and a plateau regime was found at CNT loads of above 2 wt\% in PP composites with CNT and PP based maleic anhydride as compatibilizer [48]. It should be commented that the frequency range analyzed in those investigations is, at low frequency, at least one decade shorter than the one here accessible because of the joint measurements (small-amplitude oscillatory shear and stress relaxation) performed.

The Al20 and Al30 nanocomposites could not be evaluated at the temperature interval at which the previous composites have been tested. These two materials behave much more rigidly. Measurements at $200{ }^{\circ} \mathrm{C}$ and $210{ }^{\circ} \mathrm{C}$ were performed in Al20 and Al30 but master curves could not be built since these nanocomposites did not exhibit a thermorheologically simple behavior at those temperatures. These two hybrids show a typical highly elastic rubbery behavior, with almost constant values of $\mathrm{G}^{\prime}$ and $\mathrm{G}^{\prime \prime}$, all over the frequency window covered by the rheology measurements.

Figure 2 presents the dynamic viscosity curves at $200^{\circ} \mathrm{C}$ for the different hybrids with the exception of that from $\mathrm{Al} 30$ nanocomposite where the viscosity represented was that obtained at $210^{\circ} \mathrm{C}$ because rheological measurements were not possible to be carried out at lower temperatures for this $\mathrm{Al} 30$ nanocomposite. The influence that incorporation of $\mathrm{Al}$ nanoparticles exerts on the viscosity of the iPP is clearly noticeable, especially in the low 
frequency region. The incorporation of a low amount of Al nanoparticles in the Al1 hybrid results in a slight departure from rheological performance of the pristine polymer. The Newtonian plateau region is evidently detected in the Al1 sample, which present a viscosity slightly inferior to that found in the homopolymer. This might be associated with different factors. Selective adsorption of high molecular weight molecules has been reported to decrease viscosity in certain nanoparticle-filled polymer melts [44]. Also a possible partial degradation of the polymer matrix promoted by the Al nanoparticles may contribute to reduce the viscosity of the polymer [21].

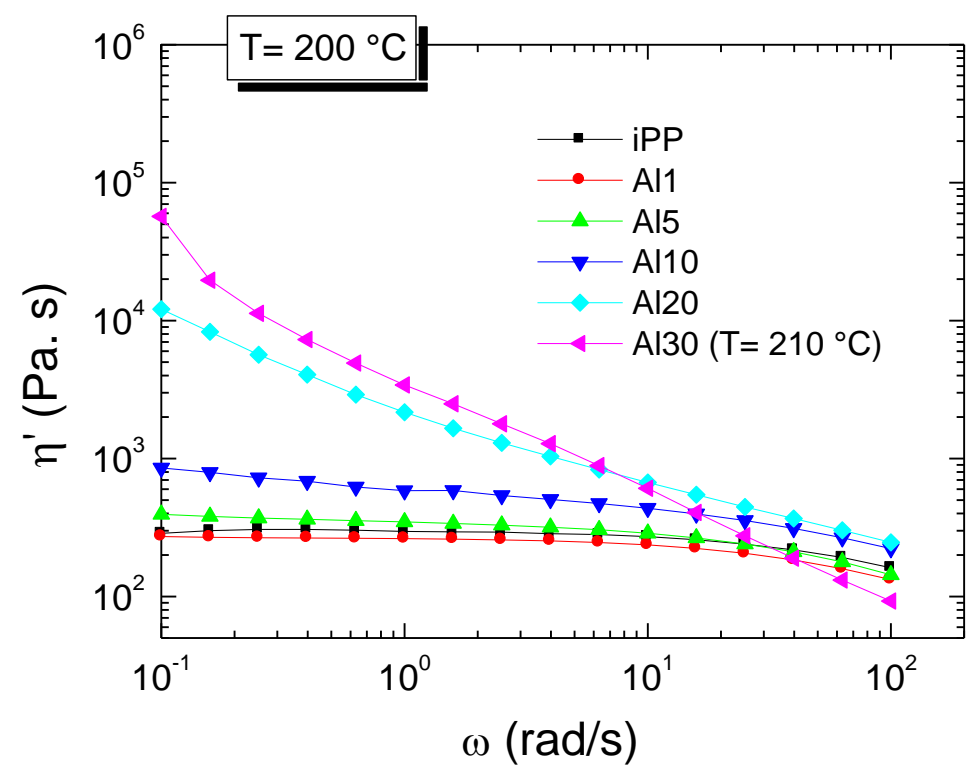

Figure 2. Dependence of dynamic viscosity on frequency for the pristine iPP and its different nanocomposites with $\mathrm{Al}$ nanoparticles at $200^{\circ} \mathrm{C}$. Experiment in the $\mathrm{Al} 30$ hybrid was performed at $\left.210^{\circ} \mathrm{C}\right)$.

The viscoelastic response at low frequencies of the Al5 hybrid turns out in a slightly higher viscosity than that exhibited by the pristine iPP homopolymer. The reinforcement role of Al nanoparticles is, here, high enough to compensate the slight decrease that can occur by incorporation of Al nanofillers and was observed in viscosity for the Al1 nanocomposite. Larger amounts of Al nanoparticles lead to progressive increases in viscosity and disappearance of the terminal Newtonian plateau, which is not longer evident even at the 
lowest frequencies. At Al contents larger than $10 \%$ in volume, typical solid-like behavior is shown with a steep rise of the viscosity as the frequency of the oscillatory deformation decreases, while the rheological behavior gets closer in the different nanocomposites to that of the neat iPP at the high frequency region. These viscosity changes are, then, similar to those exhibited by iPP composites with CNTs, although they are observed at significantly lower CNTs contents [48,49].

The phase angle $(\delta)$ can be used to evaluate the structures existing in polymers by the van Gurp-Palmen (vGP) plot $[50,51]$. The main idea of this analysis is to plot $\delta$ versus the complex modulus $\left|\mathrm{G}^{\star}\right|$. This van Gurp-Palmen representation has turned out as a useful and reliable tool for characterizing some aspects in the polymeric melts concerning their elastic or viscous nature. Figure 3 shows the results found at $190{ }^{\circ} \mathrm{C}$ for some of the specimens under study. The behavior is rather analogous for all these samples at the region of high $\left|G^{*}\right|$ but completely different for the low $\left|G^{*}\right|$ values, which correspond to the terminal zone.

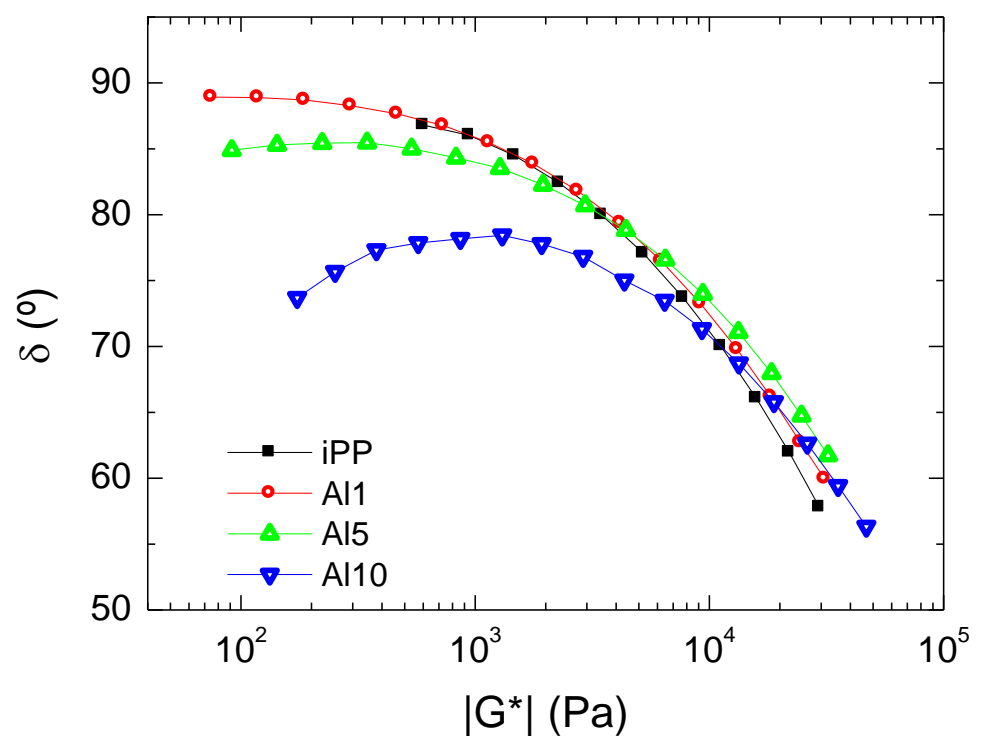

Figure 3. Phase angle $\delta$ as a function of the complex shear modulus $\left|\mathrm{G}^{*}\right|$ at $190^{\circ} \mathrm{C}$ for some of the iPP based samples. 
The iPP homopolymer curve displays the typical shape of a linear polymer. The plot of the Al1 nanocomposite is quite superimposable and phase angle is very close to $90^{\circ}$ at low $\left|G^{*}\right|$ values because its viscoelastic response under those conditions is dominated by the viscous component. The Al5 hybrid shows a reduction in the phase angle at terminal zone (low values of $\left|G^{*}\right|$ ) ascribed to the reinforcement effect from presence of Al nanoparticles that provide an elastic contribution to the iPP melt. This decrease is significantly more important in the Al10 material. Accordingly, phase angle values move away from the nearly $90^{\circ}$ observed in the pristine iPP matrix, due to incorporation of a large amount in $\mathrm{Al}$ nanoparticles that contribute to increase the elastic portion in the iPP melt because of its almost solid-like response.

The rheological behavior (and consequently processability) of the resulting iPP based materials is, thus, significantly affected by incorporation of Al nanoparticles.

\section{EMI shielding properties}

The study of the dielectric behavior of these nanocomposites demonstrated [21] that conductivity increased with frequency and was enlarged slightly with incorporation of $\mathrm{Al}$ nanoparticles at a given frequency. Nevertheless, dependence on frequency was rather similar for the nanocomposites compared with that exhibited by the neat iPP matrix. These facts indicated that all of these materials were characterized by an insulating behavior for all the Al contents analyzed. Thus, the insulator-conductor transition did not take place within this Al composition range, because electrical percolation threshold (EPT) has not been reached yet. The need of reaching the EPT for the EMI applications with adequate level of shielding (i.e. a SE $\geq 20 \mathrm{~dB}$ ) has been reported in literature [52]. Nevertheless, hybrids based on poly(vinilydene fluoride) and aluminum nanoparticles [53] showed good EMI shielding characteristics at $2 \mathrm{EHz}$ with $\mathrm{SE} \approx 20 \mathrm{~dB}$ [54] without attaining the EPT. Reason for this different behavior might be attributed to the fact that EMI characteristics are dependent on frequency interval of measurements and type of experiments. The former were performed in the $\mathrm{X}$-band frequency range and the other composites were measured at much higher 
frequency. Therefore, electrical conductivity seems not to be absolutely mandatory at some working frequencies for exhibiting a sufficient electromagnetic performance. Nevertheless, what is noticeable is that the shielding capability will be improved with the increase in the electrical conductivity $[20,52,55,56]$.

Figure 4 shows the different dependence upon Al composition observed in the improvement of relative conductivity values (conductivity exhibited by the nanocomposites referred to that found in the neat iPP matrix). A plateau-like behavior is attained at the highest $\mathrm{Al}$ compositions. This plateau involves an improvement in conductivity at $1 \mathrm{~Hz}$ of more than three decades in the Al10, Al20 and Al30 hybrids compared with the value found in the iPP matrix. As aforementioned, this rise is not high enough to trigger the insulatorconductor transition in these materials and they show an insulating behavior [21]. At the lowest Al contents, a progressive increase is seen up to the Al10, approximately one decade per sample between the Al1, Al5 and Al10 nanocomposites. At the highest Al compositions, the enlargements are considerably reduced. This distinct trend upon Al content could be probably ascribed to the formation of $\mathrm{Al}$ aggregates of large sizes that minimize the role of $\mathrm{Al}$ as shield at the highest contents. Figure 4 also displays that variations in relative conductivity are lowered as increasing frequency.

Figure 5 shows the crystalline nature of these iPP based nanocomposites. The monoclinic form is the one developed in majority under the processing conditions used, although the presence of a weak diffraction, ascribed to the (117) reflection of the $y$ form, is also observed [21]. Figure 5 points also out a noticeable decrease of the synchrotron X-ray radiation that reaches the detector, as much as the $\mathrm{Al}$ content is increased in the final material. It should be commented that this radiation is much more intense than the conventional one and that these experiments were performed in a transmission mode, fact that indicates that an absorption mechanism of radiation takes place within these nanocomposites. These results are, then, an indicator that an important increase in 
attenuation upon transmission is observed in these nanocomposites as Al content is raised, at the corresponding frequency of $2 \mathrm{EHz}(0.150 \mathrm{~nm}$ wavelength).

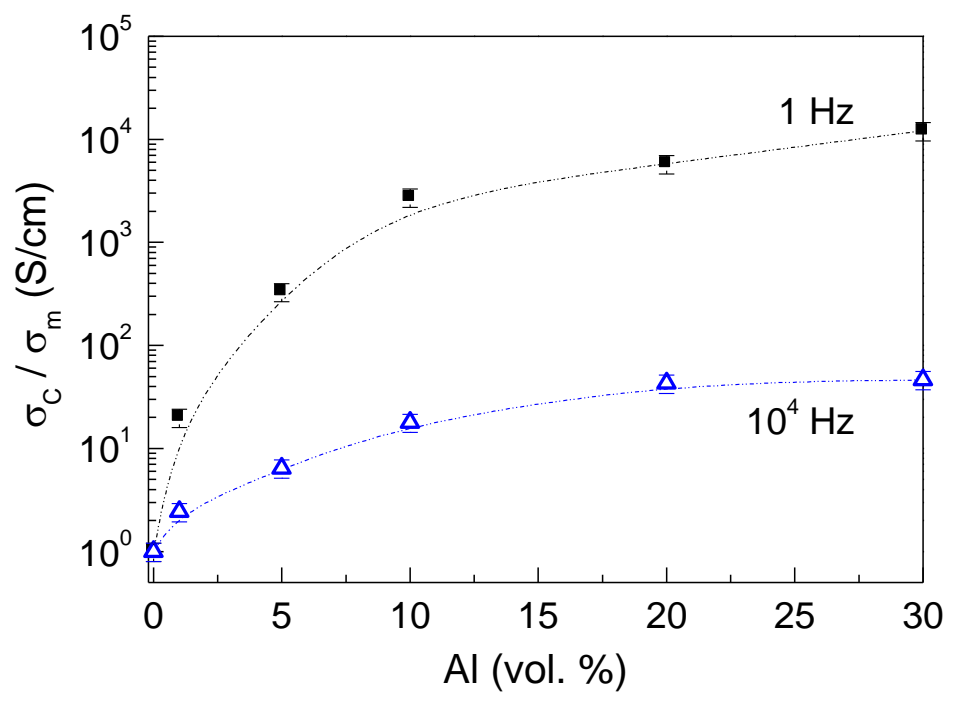

Figure 4. Relative conductivity (the one exhibited by nanocomposites, $\sigma_{\mathrm{C}}$, referred to that found in the iPP matrix, $\sigma_{\mathrm{m}}$ ) measured at $25^{\circ} \mathrm{C}$ at 1 and $10^{4} \mathrm{~Hz}$ and as a function of $\mathrm{Al}$ volume fraction.
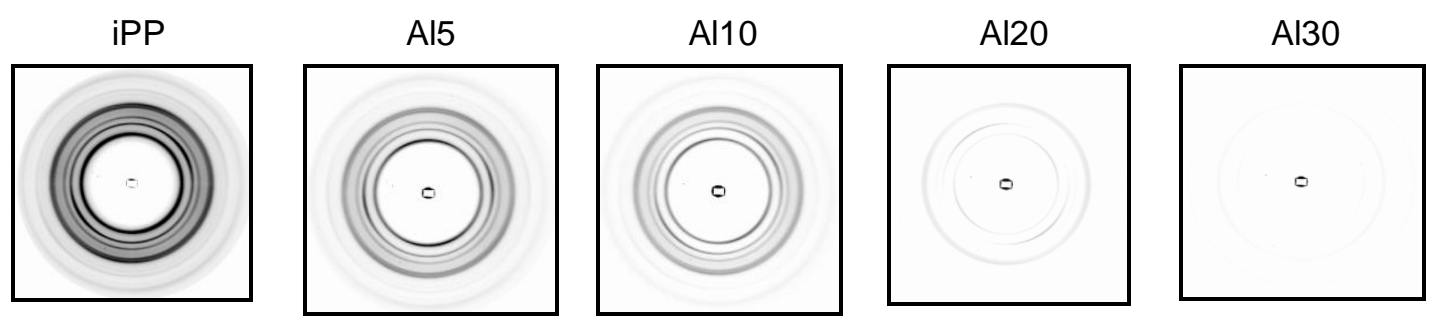

Figure 5. 2D X-ray pictures obtained with synchrotron radiation $(\lambda=0.150 \mathrm{~nm})$ at room temperature, showing variation of the Al nanoparticles effectiveness as shields for the different nanocomposites.

Shielding effectiveness (SE) achieved in the real environment, i.e., the attenuation of the electromagnetic field produced by the shield, is the parameter used to estimate the effect. It is defined [3] as the ratio of radiation transmitted in presence or absence of an absorber material, $E_{0}$ and $E_{1}$, respectively. This is expressed in decibels $(d B)$ by the formula: 


$$
S E=20 \log \left(E_{0} / E_{1}\right)
$$

These values of SE deduced from the experiments in Figure 5 will be discussed below.

The actual attenuation for any EMI shielding material is as well strongly dependent on the frequency of the incident radiation, so that shielding processes are rarely equally effective across wide ranges of electromagnetic radiation frequencies common in real life. Therefore, additional electromagnetic radiations were also tested (frequencies ranging from 7 to $15 \mathrm{GHz}$ ), as aforementioned in the Experimental Section.

Figure 6 shows EMI shielding effectiveness from attenuation upon reflection for the different nanocomposites placed on a metal layer. It is clearly observed a loss of reflectivity with relation to the incident radiation in the nanocomposites compared with the almost constant response, close to zero, exhibited by the pristine iPP matrix in the whole frequency range analyzed. It should be commented that Al20 and Al30 materials were not available for this study since obtainment of a film with the appropriate size $\left(25 \times 25 \mathrm{~cm}^{2}\right.$ as aforementioned in the Experimental Part) was not possible because of their very high viscosity values and the subsequent difficulty for their processing.

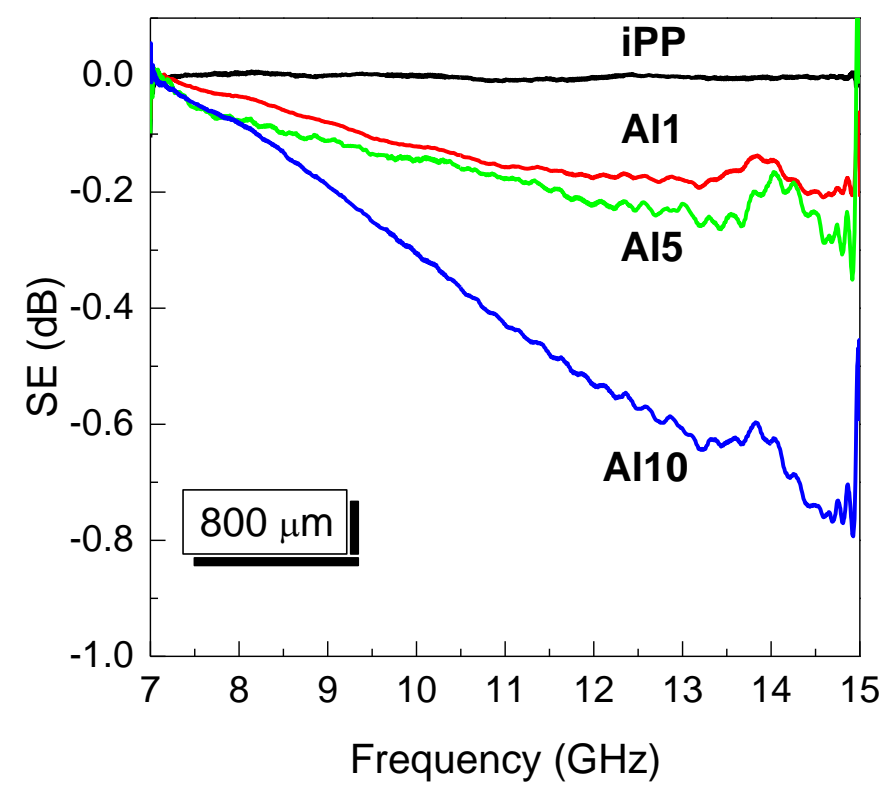

Figure 6. Return loss of the reflection coefficient $\left(\mathrm{R}_{0}\right)$ versus frequency for iPP and different nanocomposites at $800 \mu \mathrm{m}$ of distance from the metal layer. 
Results depicted in Figure 6 prove that Al10 nanocomposite presents the best shielding behavior, from the suitable hybrids. This response should be understood in terms of composite dielectric constant. The lowest Al contents are not sufficient to influence the composite electromagnetic properties and they do not allow reaching the maximum reduction. It should be considered that to be a good attenuator in this microwave frequency range it should not only absorb the energy at the microwave range but it must also have a low reflectivity. The electrical characteristics of the Al10 composite are the superior concerning that reflectivity.

The Al10 nanocomposite has been, consequently, selected to further examine the effect of distance between the nanocomposite film and the metal layer because of its better response. Thus, EMI properties have been evaluated at different distances. In this case, a "glaspack" sheet, of width between 0 and $800 \mu \mathrm{m}$, has been placed between metal and composite sample. Figure 7 represents the reduction in reflectivity that the Al10 nanocomposite undergoes with distance. In order to understand this feature the following considerations should be done. The electromagnetic radiation is incident on the composite slab in the performed experiments. It goes through it and the dielectric "glaspack", being reflected by the metallic surface. The reflection coefficient depends on the composite impedance and on distance, $x$, from the composite to the metal [57]. This reflection coefficient, $\mathrm{R}_{0}$, at $x=0$ becomes:

$$
R_{0}=\frac{\left(r_{12}+r_{23} e^{-2 j \gamma_{2} d_{2}}\right)}{\left(1+r_{12}+r_{23} e^{-2 j \gamma_{2} d_{2}}\right)}
$$

where $Y_{2}$ is the wave vector for the composite; and, $r_{12}=\left(Z_{2}-Z_{1}\right) /\left(Z_{2}+Z_{1}\right), Z_{1}$ and $Z_{2}$ being the impedance of the vacuum and the composite, respectively. The $r_{23}$ parameter verifies:

$$
r_{23}=\frac{\left(2 j \sin \gamma_{3} d_{3} Z_{3}-2 \cos r_{23} \gamma_{3} d_{3} Z_{2}\right)}{\left(2 j \sin \gamma_{3} d_{3} Z_{3}+2 \cos \gamma_{3} d_{3} Z_{2}\right)}
$$

where $Z_{3}$ is the impedance of the dielectric comprised between the composite sheet and the metallic surface and $\gamma_{3}$ is its wave vector. It is important to remark that for loss free media the impedances, $Z_{i}$, and $r_{12}$ are real, whereas $r_{23}$ is a complex number with modulus unity, $r_{23}=$ 
$\mathrm{e}^{-\mathrm{j} \zeta}$ and $\left|R_{0}\right|$ is minimum when $\zeta+2 \gamma_{2} d_{2}=\pi$ and $\zeta$ is given by $\zeta=-2 \tan ^{-1}\left(Z_{3} / Z_{2}\right.$ tan $\left.\gamma_{3} d_{3}\right)$, where $Z_{2}$ and $Z_{3}$ are the impedances for the composites and dielectric, respectively, and $d_{2}$ and $d_{3}$ are the thickness of the composite and the dielectric sheet, respectively). This rule can be used as a guide for an approximate control of frequency of the maximum attenuation, as a function of composite and dielectric distances and impedances. At a certain composite impedance (or dielectric constant) the higher the distance is between metal and composite, the lower is the frequency associated with the minimum reflectivity.

The performed experiments have demonstrated this behavior for the composites under study. The attenuation level goes from $-0.1 \mathrm{~dB}$ to $-0.75 \mathrm{~dB}$ for $15 \mathrm{GHz}$ because of the composite impedance (see Figure 7).

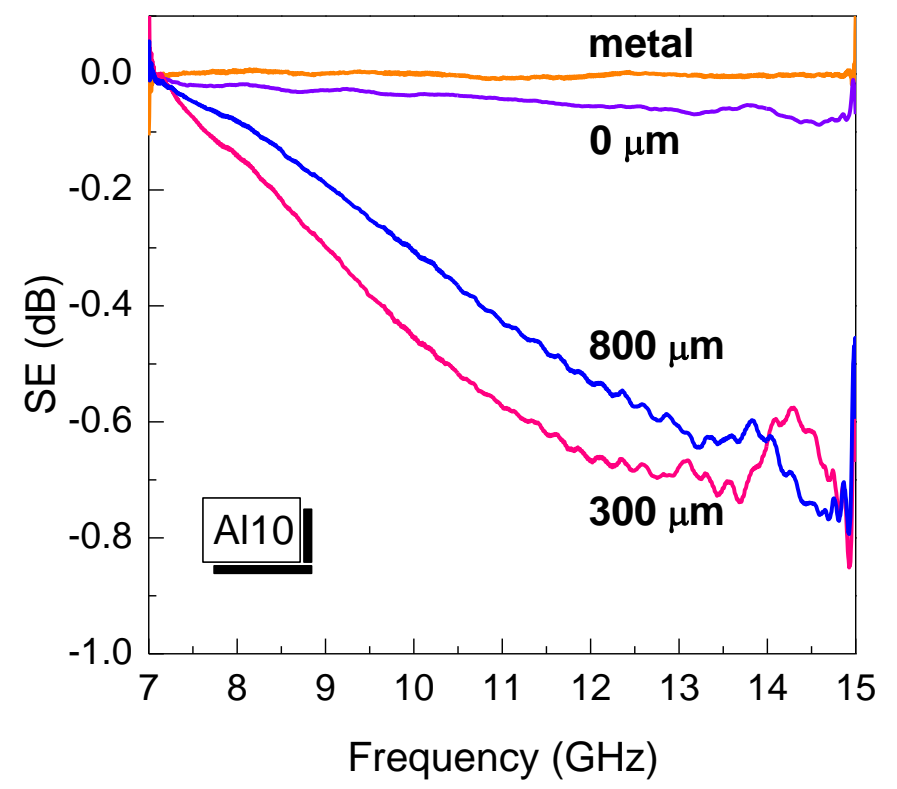

Figure 7. Return loss of the reflection coefficient $\left(R_{0}\right)$ versus frequency of Al10 nanocomposite at different distances from the metal layer.

As aforementioned, EMI shielding response is strongly dependent on the frequency of the incident radiation. Measurements performed at $2 \mathrm{EHz}$ and at the microwave range have shown the behavior as shields of these materials by absorption mechanisms. The EMI characteristics have been also checked within the infrared interval (see Figure 8) and the results have been compared with that previously obtained at $2 \mathrm{EHz}$. This infrared 
determination has been carried out from Attenuated Total Reflectance (ATR) spectra, considering the absorbance values of the plateau located at wavenumber region ranging from 3200 to $3400 \mathrm{~cm}^{-1}$, i.e., at $100 \mathrm{THz}$ as a measure of the shield capacity. Figure 8 shows the estimated shielding effectiveness at $100 \mathrm{THz}$ together with the spectral zone taken into account (see inset). The overall behavior observed is rather similar at these two frequencies, $100 \mathrm{THz}$ and $2 \mathrm{EHz}$, and an increase in SE is seen as Al nanoparticle content is enlarged in the nanocomposites. The Al1 and Al5 materials display only slight differences between them, probably due to the non optimal efficiency of small Al amounts at those specific frequencies. These results corroborate the ones achieved at the other frequency ranges explored, i.e. the improvements in the shielding capabilities with the Al incorporation. Therefore, these ATR spectra can be also utilized in these metal/polymer systems as a fast tool to learn on their capacity as shields.

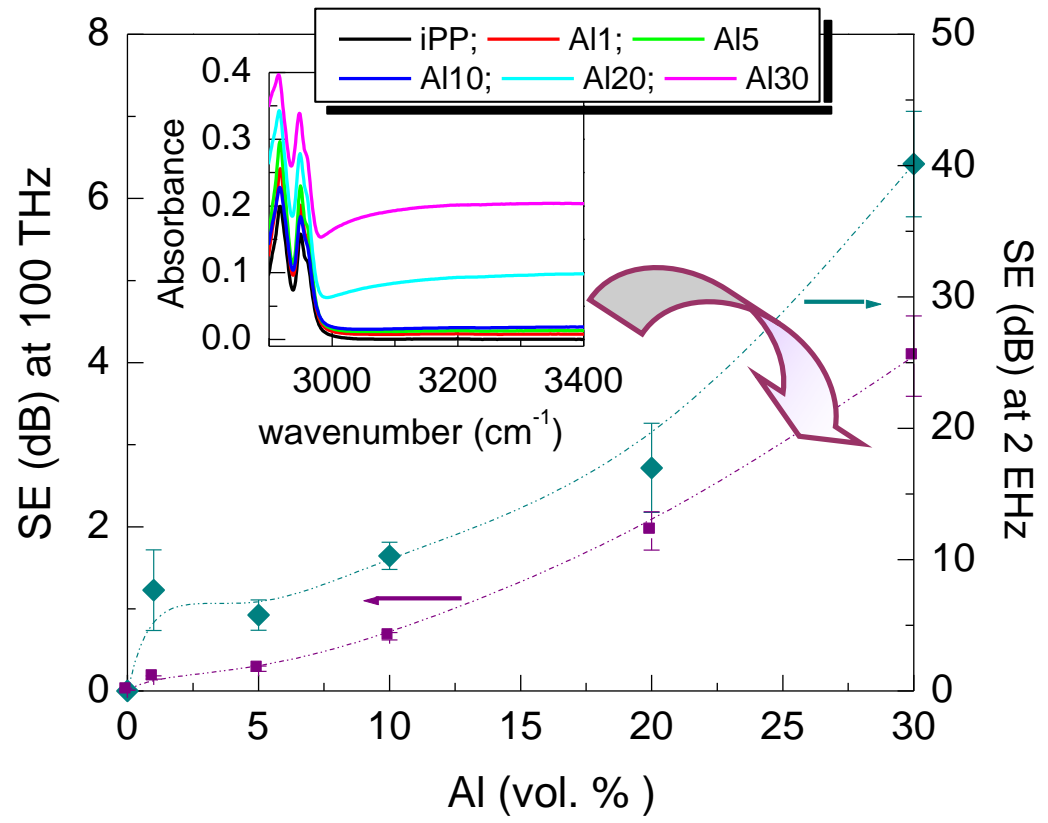

Figure 8. Comparison of the shielding effectiveness estimated at $100 \mathrm{THz}$ (left axis) and at 2 $\mathrm{EHz}$ (right axis) from infrared and X-ray electromagnetic radiations, respectively. Inset: Absorbance values obtained from the FTIR-ATR spectra, at room temperature, of iPP and the distinct hybrids with different contents of Al nanoparticles, in the selected wavenumber interval. 


\section{CONCLUSIONS}

Nanocomposites based on isotactic polypropylene (iPP) and different content of Al nanoparticles have been proposed as inert, low-cost and lightweight materials with shielding characteristics against electromagnetic interference and radiation.

Incorporation of Al nanoparticles significantly affects the rheological behavior and, consequently, processability of the resulting iPP based materials. Combination of dynamic experiments with stress relaxation measurements has allowed spreading frequency scale in several orders of magnitude. It is, then, noticeable that time-temperature superposition principle is valid up to an $\mathrm{Al}$ content of $10 \%$ in volume but a thermo-rheological complex behavior, not allowing a master curve construction, is observed at higher Al amounts.

Moreover, pristine iPP matrix shows the expected frequency dependence in the terminal region while the Al1 hybrid already exhibits small variations in dissipation modes. A more evident departure is shown in the Al5 nanocomposite because of the formation of a nanofiller-like network and the attainment of the rheological percolation threshold, as deduced from dependence of G' and G" on frequency. This elastic reinforcement effect of Al particles is more clearly defined in the Al10 material and, the melt behaves much more rigidly in the nanocomposites with the highest contents, Al20 and Al30, which display a typical highly elastic rubbery behavior.

EMI shielding effectiveness from attenuation upon reflection at a bandwidth range from 7 to $15 \mathrm{GHz}$ has shown a loss of reflectivity with relation to the incident radiation for the different nanocomposites. The Al10 nanocomposite presents the best shielding behavior, this response being understood in terms of composite dielectric constant.

Infrared spectroscopy using attenuated total reflection (ATR) has been proved as fast approach for knowledge of EMI shielding capability, at wavenumber region from 3200 to $3400 \mathrm{~cm}^{-1}$. The shielding effectiveness at $100 \mathrm{THz}$ increases as Al nanoparticle content does in the nanocomposites, these results being in a good agreement with those attained at $2 \mathrm{EHz}$ and at the microwave region. 


\section{Acknowledgements}

The financial support of AEI/FEDER, UE (project MAT2016-79869-C2-1-P) is acknowledged. The synchrotron work leading to these results received funding from the European Community's Seventh Framework Programme (FP7/2007-2013) under grant agreement $n^{\circ} 312284$.

\section{References}

[1] C.R. Paul, Introduction to Electromagnetic Compatibility, Second ed., John Wiley \& Sons Inc., New Jersey, 2006.

[2] K. Malaric, EMI Protection for Communication Systems, Artech House, 2010.

[3] X.C. Tong, Advanced Materials and Design for Electromagnetic Interference Shielding, CRC Press, New York, 2009.

[4] S. Celozzi, G. Lovat, R. Araneo, Electromagnetic Shielding in Wiley Encyclopedia of Electrical and Electronics Engineering, John Wiley \& Sons, Inc., Hoboken, New Jersey, 2008.

[5] ICNIRP, Exposure to High Frequency Electromagnetic Fields, Biological Effects and Health Consequences (100 kHz-300 GHz), ICNIRP, Munich, 2009.

[6] ICNIRP, Health Physics, 74 (1998) 494-522.

[7] D.D.L. Chung. Materials for electromagnetic interference shielding. J. Mater. Eng. Perform. 9 (2000), 350-4.

[8] R.B. Schulz, V.C. Plantz, D.R. Brush. Shielding theory and practice. Electromagnetic compatibility. IEEE Trans. 30 (1988), 187-201.

[9] E.F. Knott, J.F. Schaeffer, M.T. Tuley, Radar Cross Section, SciTech Publishing, Inc. Raleigh, NC, 2004.

[10] R.B. Thompson, VV. Ginzburg, M.W. Matsen, A.C. Balazs, Predicting the Mesophases of Copolymer Nanoparticle Composites, Science 292 (2001) 2469-2472.

[11] M.E. Mackay, A. Tuteja, P.M. Duxbury, C.J. Hawker, B. Van Horn, Z. Guan, G. Chen, R.S. Krishnan, General Strategies for Nanoparticle Dispersion, Science 311 (2006) 1740-1743.

[12] S.C. Warren, F.J. DiSalvo, U. Wiesner, Nanoparticle-tuned assembly and disassembly of mesostructured silica hybrids, Nat Mater 6 (2007) 156-161. 
[13] M.E. Mackay, T.T. Dao, A. Tuteja, D.L. Ho, B. Van Horn, H.C. Kim, C.J. Hawker, Nanoscale effects leading to non-Einstein-like decrease in viscosity, Nat Mater 2 (2003), 762-766.

[14] A. Bansal, H. Yang, C. Li, K. Cho, B.C. Benicewicz, S.K. Kumar, L.S. Schadler, Quantitative equivalence between polymer nanocomposites and thin polymer films, Nat Mater 4 (2005) 693698.

[15] R.A. Narayanan, P. Thiyagarajan, S. Lewis, A. Bansal, L.S. Schadler, L.B. Lurio, Dynamics and internal stress at the nanoscale related to unique thermomechanical behavior in polymer nanocomposites, Phys. Rev. Lett. 97 (2006) 075505/1-075505/4.

[16] V. Graves, Polypropylene: A Commodity Plastic Reaches Record Highs in 1994 Production, Modern Plastics Encyclopedia, McGraw-Hill 1996. "Polypropylene: High Price/Performance Ratio Provides Growth", Modern Plastic, McGraw-Hill, 1997, vol 74.

[17] M.L. Cerrada, C. Serrano, M. Sánchez-Chaves, M. Fernández-García, M.A. de Andrés, R.J. Riobóo, F. Fernández-Martín, A. Kubacka, M. Ferrer, M. Fernández-García, Biocidal Capability Optimization in Organic Inorganic Nanocomposites Based on Titania, Environ. Sci. Techn. 43 (2009) 1630-1634.

[18] A. Kubacka, M. Ferrer, M.L. Cerrada, C. Serrano, M. Sánchez-Chaves, M. Fernández-García, A. de Andrés, R.J. Jiménez-Riobóo, F. Fernández-Martín, M. Fernández-García, Boosting TiO2anatase antimicrobial activity: polymer-oxide thin films, Appl. Catal. B-Environ. 89 (2009) 441447.

[19] A. Kubacka, M. Ferrer, M. Fernández-García, C. Serrano, M.L. Cerrada, M. Fernández-García, Tailoring Polymer-TiO2 properties by presence of metal $(\mathrm{Ag}, \mathrm{Cu}, \mathrm{Zn})$ species: Optimization of Antimicrobial Properties, Appl. Catal. B-Environ. 104 (2011) 346-352.

[20] J. Arranz-Andrés, E. Pérez, M.L. Cerrada, Nanocomposites based on Isotactic PolypropyleneCopper Nanoparticles as Electromagnetic Shields, Sci. Advan. Mater. 5 (2013) 1524-1532.

[21] J. Arranz-Andrés, E. Pérez, M.L. Cerrada, Lightweight Nanocomposites based on Polypropylene and Aluminum Nanoparticles and their Shielding Capability to lonizing Radiation, IEEE T. Nanotechnol. (TNANO) 13 (2014) 502-509.

[22] M.H. Al-Saleh, U. Sundararaj, Electromagnetic interference (EMI) shielding effectiveness of PP/PS polymer blends containing high structure carbon black, Macromol. Mat. Eng. 293 (2008) 621. 
[23] M.H. Al-Saleh, U. Sundararaj, X-band EMI shielding mechanisms and shielding effectiveness of high structure carbon black/polypropylene composites, J. Phys. D: Appl. Phys. 46 (2013) No. 035304.

[24] M.H. Al-Saleh, U. Sundararaj, Electromagnetic interference shielding mechanisms of CNT/polymer composites, Carbon 47 (2009) 1738-1746.

[25] J.-M. Thomassin, I. Huynen, R. Jerome, Ch. Detrembleur, Functionalized polypropylenes as efficient dispersing agents for carbon nanotubes in a polypropylene matrix: application to electromagnetic interference (EMI) absorber materials, Polymer 51 (2010) 115-121.

[26] A.J. Paleo, F.W.J. Van Hattum, J. Pereira, J.G. Rocha, J. Silva, V. Sencadas, S. LancerosMéndez, The piezoresistive effect in polypropylene-carbon nanofibre composites obtained by shear extrusion, Smart Mater. Struct. 19 (2010) No. 065013.

[27] V. Panwar, J.O. Park, S.H. Park, S. Kumar, R.M. Mehra, Electrical, Dielectric, and Electromagnetic Shielding Properties of Polypropylene-Graphite Composites. J. Appl. Polym. Sci. 115 (2010) 1306-1314.

[28] Q. Yu, F. Wan, W. Gao, J. Li, S. Guo, Structure and electromagnetic interference shielding properties of microlayered PPGr/PP composites, Polym. Mater. Sci. Eng. 28 (2012) 30-33.

[29] S.T. Tan, M.Q. Zhang, M.Z. Rong, H.M. Zeng, F.M. Zhao, Properties of metal fibre filled thermoplastics as candidates for electromagnetic interference shielding, Polym. Polym. Compos. 9 (2001) 257-262.

[30] N. A. Pratten, Journal of Materials Science 16, 1737 (1981).

[31] J.D. Ferry, Viscoelastic properties of polymers, New York: John Wiley and Sons 1980.

[32] Y. Wang, X. Jing, Intrinsically conducting polymers for electromagnetic interference shielding, Polym. Advan. Technol. 16 (2005) 344-351.

[33] P. Marin, D. Cortina, A. Hernando, Electromagnetic waves absorbing material based on magnetic microwires, IEEE Transactions on Magnetics 44 (2008) 3934-3937.

[34] G.D. Smith, D. Bedrov, I. Li, O. Byutner, A molecular dynamics simulation study of the viscoelastic properties of polymer nanocomposites, J. Chem. Phys. 117 (2002) 9478-9489.

[35] Q. Zhang, L.A. Archer, Poly(ethylene oxide)/silica nanocomposites: Structure and rheology. Langmuir 18 (2002) 10435-10442. 
[36] A.S. Sarvestani, E. Jabbari, Modeling and experimental investigation of rheological properties of injectable poly(lactide ethylene oxide fumarate)/hydroxyapatite nanocomposites, Biomacromolecules 7 (2006) 1573-1580.

[37] B. Erman, Structures and Properties of Rubber-like Networks, Oxford University Press: New York, 1997, p 370.

[38] M.I. Aranguren, E.M. Mora, J.V. Degroot, C.V. Macosko, Effect of reinforcing fillers on the rheology of polymer melts. J. Rheol. 36 (1992) 1165-1182.

[39] N. Jouault, P. Vallat, F. Dalmas, S. Said, J. Jestin, F. Boue, Well-Dispersed Fractal Aggregates as Filler in Polymer-Silica Nanocomposites: Long-Range Effects in Rheology, Macromolecules 42 (2009) 2031-2040.

[40] A.S. Sarvestani, Modeling the solid-like behavior of entangled polymer nanocomposites at low frequency regimes, Eur. Polym. J. 44 (2008) 263-269.

[41] D. Acierno, G. Filippone, G. Romeo, P.Russo, Dynamics of stress bearing particle networks in poly(propylene)/alumina nanohybrids, Macromol. Mater. Eng. 292 (2007) 347-353.

[42] H.A. Barnes, The rheology of filled viscoelastic systems: A review, Rheology Reviews 2003, British Society of Rheology (2003) 1-36.

[43] W. Bahloul, V. Bounor-Legaré, L. David, P. Cassagnau, Morphology and viscoelasticity of PP/TiO2 nanocomposites prepared by in situ sol-gel method, J. Polym. Sci., Part B: Polym. Phys. 48 (2010) 1213-1222.

[44] S. Jain, J.G.P. Goossens, G.W.M. Peters, M. van Duin, P.J. Lemstra, Strong decrease in viscosity of nanoparticle-filled polymer melts through selective adsorption, Soft Matter. 4 (2008) $1848-1854$.

[45] H.M. Hassanabadi, M. Wilhelm, D. Rodrigue, A rheological criterion to determine the percolation threshold in polymer nano-composites, Rheol. Acta 53 (2014) 869-882.

[46] C. Serrano, M.L. Cerrada, M. Fernández-García, J. Ressia, E.M. Vallés, Rheological and Structural Details of Biocidal iPP-TiO2 Nanocomposites, Eur. Polym. J. 48 (2012) 586.

[47] C. Serrano, J.A. Ressia, E.M. Vallés, M. Fernández-García, M.L. Cerrada, Interfacial Agent Effect on Rheological Response and Crystallite Characteristics in Germicide Polypropylene/Titanium Dioxide Nanocomposites, Polym. Int. 61 (2012) 1655-1665. 
[48] S.H. Lee, M.W. Kim, S.H. Kim, J.R. Youn, Rheological and electrical properties of polypropylene/MWCNT composites prepared with MWCNT masterbatch chips, Eur. Polym. J. 44 (2008) 1620-1630.

[49] M. Ganß, B.K. Satapathy, M. Thunga, R. Weidisch, P. Pötschke, D. Jehnichen, Structural interpretations of deformation and fracture behavior of polypropylene/multi-walled carbon nanotube composites, Acta Materialia 56 (2008) 2247-2261.

[50] S. Trinkle, C. Friedrich, Van Gurp-Palmen-plot: a way to characterize polydispersity of linear polymers, Rheol. Acta 40 (2001) 322-328.

[51] M.L. Cerrada, E. Pérez, R. Benavente, J. Ressia, C. Sarmoria, E.M. Vallés, Gamma Polymorph and Branching Formation as Inductors of Resistance to Electron Beam Irradiation in Metallocene Isotactic Polypropylene, Polym. Degrad. Stab. 95 (2010) 462-469.

[52] M. H. Al-Saleh. Electrical, EMI shielding and tensile properties of PP/PE blends filled with GNP:CNT hybrid nanofiller. Synthetic Metals 217 (2016) 322-330.

[53] J. Arranz-Andrés, N. Pulido González, P. Marín, A. Aragón, M.L. Cerrada. Electromagnetic Shielding Features in Lightweight PVDF Aluminum Based Nanocomposites, Progress in Electromagnetics Research B 48 (2013) 175-196.

[54] J. Arranz-Andrés, N. Pulido-González, C. Fonseca, E. Pérez, M.L. Cerrada, Lightweight Nanocomposites based on Poly(Vinylidene Fluoride) and Al Nanoparticles: Structural, Thermal and Mechanical Characterization as well as their EMI Shielding Capability, Mater. Chem. Phys. 142 (2013) 469-478.

[55] J. Arranz-Andrés, E Pérez, M.L. Cerrada, Hybrids based on Poly(Vinylidene Fluoride) and Cu Nanoparticles: Characterization and EMI Shielding, Eur. Polym. J. 48 (2012) 1160-1168.

[56] J. Arranz-Andrés, M. L. Cerrada. Conductive Poly(vinylidene fluoride)/Copper Hybrids: Mechanical Response and Percolation Threshold. Sci. Advan. Mater. 5 (2013) 1-9.

[57] A.G. Gorriti, P. Marin, D. Cortina, A. Hernando, Microwave attenuation with composite of copper microwires, J. Magn. Magn. Mater. 322 (2010) 1505-1510. 